\title{
Méthodes de datation en Sciences de la Terre
}

\author{
Nathalie FAGEL ${ }^{1}$ \\ Département de géologie, Université de Liège \\ Quartier Agora, B18, allée du six Août 14 \\ 4000 Liège 1, Belgique
}

\section{Introduction}

La radioactivité des roches ou sédiments est liée à la désintégration spontanée d'un nucléide instable en un nucléide stable avec émission d'une radiation. Au fil du temps, l'isotope parent radioactif ou radioisotope va se désintégrer progressivement en un isotope stable. L'abondance relative entre l'isotope parent et l'isotope fils va donc évoluer en fonction du temps. Le couple parent/fils définit ainsi un chronomètre ou géochronomètre permettant de dater les roches (Table 1). Chaque couple parent/fils est représenté par un temps caractéristique ou période de demi-vie. Ce temps caractéristique correspond à l'intervalle de temps nécessaire pour que la moitié des atomes parentaux radioactifs se désintègrent. Au bout de 5 à 10 périodes de demi-vies, la quantité d'atomes parentaux devient trop faible pour être mesurée et le chronomètre n'est plus utilisable. Chaque géochronomètre permet de dater un intervalle de temps spécifique. Les géochronomètres à longues périodes de demi-vie, de 100 millions à quelques dizaines de milliards d'années, permettent de dater des roches du Précambrien. Les géochronomètres à courtes périodes de demi-vies, de 1 million à quelques milliers d'années, permettent de dater des roches du Quaternaire.

Table 1 : Les principaux géochronomètres utilisés en Sciences de la Terre et leur domaine temporel d'utilisation (repris de Walker 2005, Bradley 1999).

\begin{tabular}{|c|c|c|c|}
\hline $\begin{array}{l}\text { Isotope } \\
\text { parent }\end{array}$ & Isotope fils & $\begin{array}{c}\text { Période de } \\
\text { demi-vie }\end{array}$ & Origine \\
\hline $\mathrm{U}-238$ & $\mathrm{~Pb}-206$ & $4.5110^{9}$ années & \multirow{6}{*}{$\begin{array}{l}\text { Nucléides primordiaux présents dans les } \\
\text { roches terrestres }\end{array}$} \\
\hline $\mathrm{U}-235$ & $\mathrm{~Pb}-207$ & $71310^{8}$ années & \\
\hline Th-232 & $\mathrm{Pb}-208$ & $13.910^{9}$ années & \\
\hline $\mathrm{Rb}-87$ & Sr-87 & $48.810^{9}$ années & \\
\hline $\mathrm{K}-40$ & Ar-39 & $1.3110^{9}$ années & \\
\hline Sm-147 & $\mathrm{Nd}-143$ & $10610^{9}$ années & \\
\hline U-234 & & $24810^{3}$ années & \multirow[t]{5}{*}{ Série de désintégration de l'uranium } \\
\hline Th-230 & & $7510^{3}$ années & \\
\hline $\mathrm{Pa}-231$ & & $34.310^{3}$ années & \\
\hline Ra-226 & & $1.610^{3}$ années & \\
\hline $\mathrm{Pb}-210$ & & 22 ans & \\
\hline Be-10 & & $1.510^{6}$ années & \multirow{2}{*}{$\begin{array}{l}\text { Cosmonucléides formés dans la haute } \\
\text { atmosphère }\end{array}$} \\
\hline $\mathrm{C}-14$ & & 5730 années & \\
\hline
\end{tabular}

Les géochronomètres peuvent être classés en 3 catégories selon leur origine: 1) les nuclides primordiaux naturellement présents dans les roches terrestres ; 2) les nuclides issus des séries de désintégration de l'uranium et du thorium et ; 3) les cosmonuclides produits dans la haute atmosphère. Les nuclides primordiaux et ceux issus des séries de désintégration de l'uranium

\footnotetext{
${ }^{1}$ Nathalie.Fagel@uliege.be
} 
et du thorium sont liés aux éléments radioactifs présents dans les roches terrestres. Par contre, les cosmonuclides ont une origine atmosphérique.

Dans la suite du texte, nous avons choisi d'expliquer plus en détail une méthode de datation parmi les plus utilisée en géologie. Il s'agit de la méthode de datation au radiocarbone.

\section{Principes de base de la méthode de datation au radiocarbone}

Le carbone comprend 3 isotopes, i.e. nucléide d'un élément chimique caractérisé par un nombre spécifique de neutrons. Le carbone 12 est le plus abondant, il représente $98.9 \%$ du carbone. Le carbone 13 représente $1.1 \%$ et le carbone 14 une infime fraction $\left(1 / 10^{10} \%\right)$. Le carbone 12 et le carbone 13 sont stables. Par contre, le carbone 14 est instable ou radioactif, il se désintègre en une forme stable d'azote $\left({ }^{14} \mathrm{~N}\right)$.

Le carbone 14 ou radiocarbone est formé par l'interaction des rayons cosmiques avec l'azote présent dans l'atmosphère. L'azote 14 capture un neutron et perd un proton pour donner un atome de carbone 14 . Cet atome va s'oxyder en dioxyde de carbone $14\left({ }^{14} \mathrm{CO}_{2}\right)$. Celui-ci va se mélanger au $\mathrm{CO}_{2}$ atmosphérique et entrer dans le cycle global du carbone. $\mathrm{Le}{ }^{14} \mathrm{CO}_{2}$ va être assimilé par les plantes pour la photosynthèse et par les animaux via l'ingestion de végétaux. La majorité du carbone 14 est incorporé dans l'océan sous forme de carbonates dissouts qui seront intégrés dans la productivité marine. Le contenu en carbone 14 des organismes vivants marins et terrestres reste en équilibre avec l'atmosphère. La désintégration du carbone 14 dans la biosphère sera compensée par le prélèvement du carbone 14 atmosphérique. Le réservoir global de carbone reste approximativement constant en fonction du temps. Cependant à la mort des organismes, cet équilibre s'interrompt. Le carbone 14 va diminuer avec une période de demi-vie de 5730 ans et produire de l'azote 14 qui va se mélanger à l'azote atmosphérique.

La mesure du contenu en carbone 14 résiduel d'un matériel fossile, comparée à un matériel standard, sera indicative de l'âge de la mort de l'organisme. La méthode de datation du radiocarbone est applicable à de nombreux matériaux géologiques contenant du carbone : fragment de bois ou de végétal, charbon, ossement, coquille carbonatée ou sédiment. Le taux de désintégration du carbone 14 est exponentiel (Figure 2). Après 5730 ans, la matière organique morte ne contient plus que $50 \%$ de sa teneur initiale en carbone 14, avec une activité de 7.8 désintégrations par minute et par gramme de carbone. Après 2 périodes $(11460$ ans), l'activité diminue à 3.9 désintégration par minute et par gramme. Après 40.000 ans, il reste moins de $1 \%$ de la teneur initiale de radiocarbone. Globalement le radiocarbone permet de dater des matériaux géologiques de moins de 50.000 ans d'âge. 


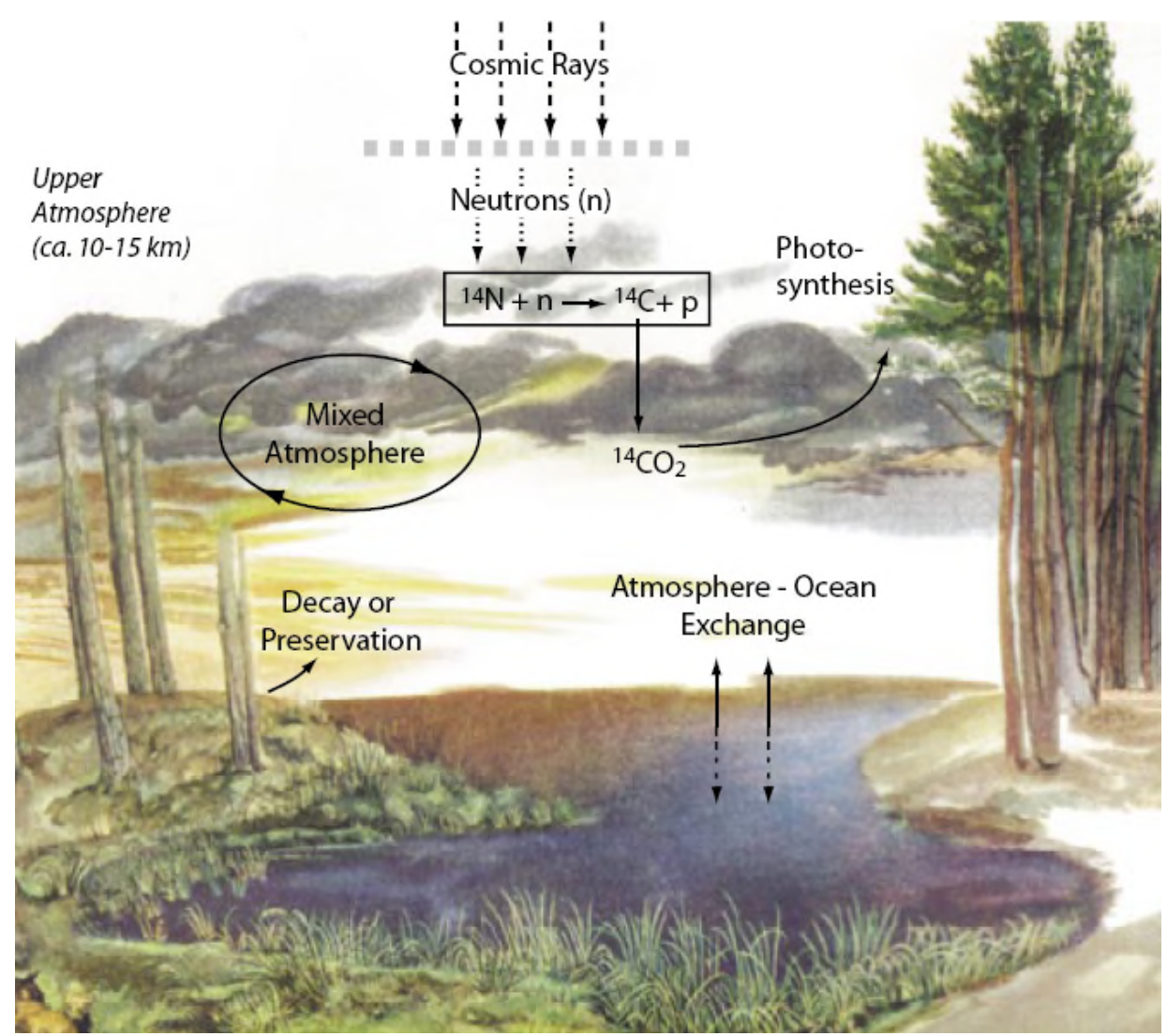

Figure 1 : Production et cycle du carbone 14. (Repris de Hajdas et al., 2006).

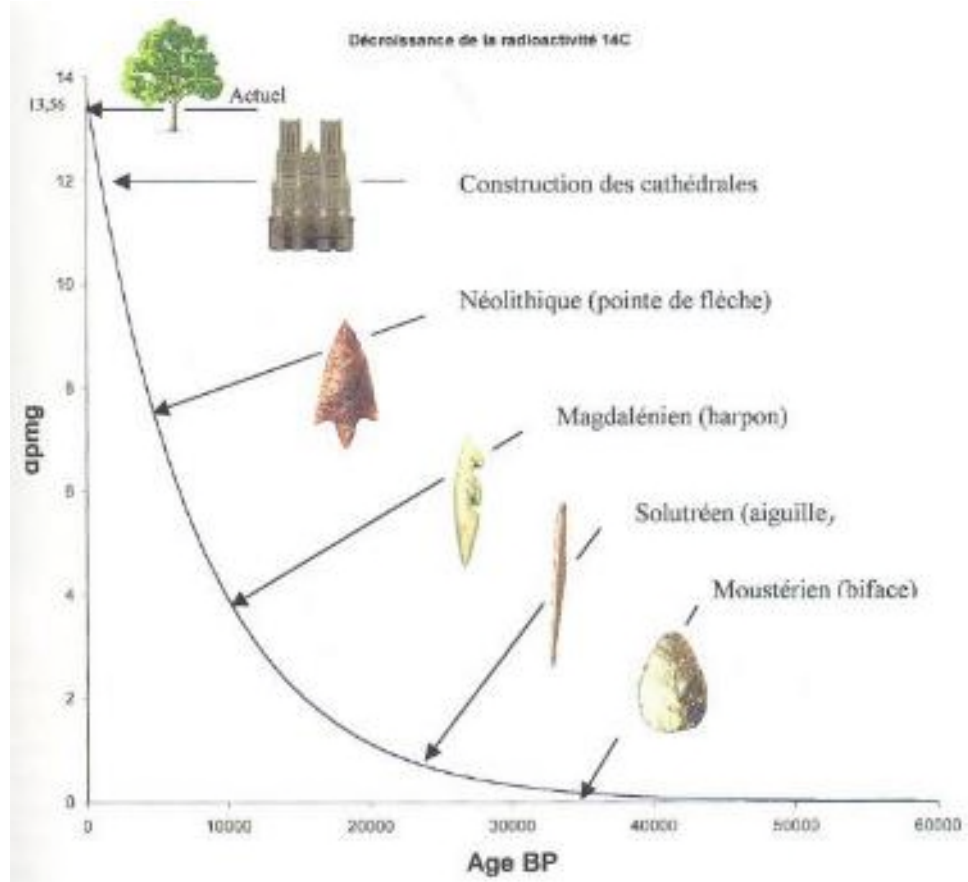

Figure 2 : Courbe de désintégration du radiocarbone en fonction du temps. (Repris de Oberlin, 2005). 


\section{Correction et calibration des âges radiocarbone}

Plusieurs sources d'erreur sont à considérer et doivent être corrigées pour traduire un âge radiocarbone en un âge calendaire. La méthode implique que les 3 conditions suivantes soient respectées :

\subsection{Budget du radiocarbone}

La discordance entre les âges obtenus par comptage des cernes annuels d'arbres et leur âge radiocarbone a permis de réfuter la première condition. Cette observation implique que le taux de production du radiocarbone dans l'atmosphère n'est pas constant mais présente des variations. Les causes de ces variations restent controversées. Elles sont probablement influencées par l'activité solaire et/ou par le champ magnétique terrestre qui modulent le flux de rayons cosmiques et donc la production de radiocarbone. Par exemple davantage de cosmonucléides seront formés lors d'une période d'activité solaire réduite comme cela s'est produit lors de l'événement froid du Petit Age Glaciaire au cours du XVI et XVII ${ }^{\text {ième }}$ siècles. Les âges radiocarbones doivent donc être calibrés pour obtenir l'âge vrai ou âge calendaire. La calibration consiste à comparer les âges radiocarbones avec des âges obtenus sur d'autres archives datées avec une résolution annuelle. Les archives les plus utilisées sont les cernes de croissance des arbres ou des coraux, les laminations sédimentaires des varves lacustres ou des concrétions carbonatées (spéléothèmes) des grottes.

En plus de ces causes naturelles, des causes artificielles sont responsables d'une diminution ou d'augmentation du radiocarbone dans l'atmosphère. Au cours de la révolution industrielle, la combustion des énergies fossiles (charbon, pétrole) apporte dans l'atmosphère du $\mathrm{CO}_{2}$ âgé, dépourvu de radiocarbone diluant la quantité de carbone 14 dans atmosphère. Au contraire, les tirs et essais nucléaires réalisés depuis une soixante d'années injectent dans l'atmosphère des produits récents qui enrichissent l'atmosphère en carbone 14. Le contenu en radiocarbone de l'atmosphère a quasi doublé dans les années 60 suite à cet effet appelé effet industriel.

\subsection{Effet réservoir}

L'homogénéisation du carbone 14 dans un réservoir n'est pas instantanée. Prenons l'exemple de l'océan. Le carbone 14 atmosphérique est incorporé dans les eaux de surfaces. Quand les eaux plongent, il n'y a plus d'équilibre avec l'atmosphère et le radiocarbone se désintègre sans être renouvelé. L'eau de mer a donc un âge apparent qui varie de quelques dizaines à quelques centaines d'années, 400 ans en moyenne, dans les eaux de surface. Dans les eaux profondes, l'âge apparent peut atteindre quelques milliers d'années. L'âge radiocarbone des organismes marins doit être corrigé de cet effet appelé effet réservoir.

Les variations de l'âge apparent des océans sont liées à la circulation océanique qui varie dans l'espace mais aussi dans le temps. Des variations temporelles dans les échanges entre l'atmosphère et l'océan influencent le budget du carbone 14 dans l'atmosphère. Ainsi un ralentissement de la circulation océanique, comme observé lors de l'événement froid du Dryas récent (il y a 12800 à 11500 ans), ralentit les échanges avec l'atmosphère. Le transfert de carbone 14 vers l'océan profond est réduit et la teneur en carbone 14 de l'atmosphère 
augmente. Le temps de mélange des océans est plus long et donc la correction de l'effet réservoir plus importante.

\subsection{Fractionnement isotopique du carbone}

Les processus biologiques et physiques sont responsables d'un fractionnement isotopique du carbone. Au niveau métabolique, les organismes n'incorporent pas les différents isotopes du carbone de la même manière. Ils ont tendance à privilégier l'isotope le plus léger du carbone ${ }^{12} \mathrm{C}$. Le taux d'incorporation varie d'une espèce à l'autre. Une plante aura une plus faible activité en carbone 14 que l'atmosphère ambiante. L'âge carbone 14 mesuré sur le reste fossile végétal sera donc vieilli par rapport à l'âge réel. Par contre, l'effet inverse sera observé pour des fossiles marins. En effet, l'océan incorpore davantage d'isotope lourd du carbone lors de la dissolution du carbone atmosphérique. Cet enrichissement en radiocarbone sera ensuite enregistré dans les organismes marins qui prélèvent les ions carbonatés des eaux pour former leur squelette. Les fossiles marins présenteront un âge rajeuni par rapport à l'âge vrai. L'erreur liée au fractionnement isotopique est corrigée en mesurant le rapport entre les isotopes stables du carbone ${ }^{13} \mathrm{C} /{ }^{12} \mathrm{C}$ de l'échantillon à dater. Ce rapport est comparé à celui d'un standard international (i.e, fossile de belemnite du Crétacé trouvé dans la formation Peedee en Caroline du sud aux Etats-Unis appelé «PDB»). La déviation par rapport à ce standard ou ${ }^{13} \delta$ s'exprime en \%o selon l'expression suivante :

$$
{ }^{13} \delta=\left[\left({ }^{13} \mathrm{C} /{ }^{12} \mathrm{C}_{\text {échantillon }}\right) /\left({ }^{13} \mathrm{C} /{ }^{12} \mathrm{C}_{\mathrm{PDB}}\right)-1\right] \times 1000
$$

Elle permet d'obtenir une valeur précise du taux de fractionnement isotopique de l'échantillon daté. En général aucune correction n'est appliquée si le ${ }^{13} \delta$ mesuré est $-25 \%$, valeur correspondant à la moyenne de la majorité du bois et des plantes terrestres. Une valeur de $30 \%$ o correspond à une déviation de $5 \%$ du rapport ${ }^{13} \mathrm{C} /{ }^{12} \mathrm{C}$ et du double (soit $10 \%$ ) en carbone 14. En effet, par convention, les laboratoires de datation radiocarbone considèrent que l'enrichissement en radiocarbone est double par rapport au carbone 13. Cela représente une correction de 83 ans sur l'âge radiocarbone.

\section{Conclusion}

La datation des roches et des sédiments est essentielle en géologie pour comprendre et retracer l'histoire de la Terre et reconstruire les paléoenvironnements. Les géochronomètres sont des outils très puissants permettant d'obtenir une datation absolue. Chaque géochronomètre possède son champ d'application, en terme de nature du matériel datable, et son intervalle de temps. L'exemple de la méthode de datation au radiocarbone montre les limites et corrections à apporter avant de traduire un âge mesuré en âge calendaire. Dans l'exposé, l'application d'autres géochronomètres sera présentée et illustrée dans le cadre de projets de recherches menés en Belgique. Les modèles d'âges obtenus sur des séquences de tourbes des Fagnes seront présentés pour illustrer la complémentarité des méthodes de datation radiocarbone et plomb 210 (Allan et al., 2013). Nous présenterons également les résultats de datations par le couple U/Th de roches carbonatées prélevées dans les grottes des 
Ardennes belges (Allan et 2015 \& 2018). Ces résultats seront combinés avec d'autres traceurs chronologiques (laminations annuelles, radiocarbone) pour démontrer la fiabilité des géochronomètres.

Quelques références pour en savoir plus....

Allan et al. 2013. Mid and late Holocene dust deposition in Western Europe: The Misten peat bog (Hautes Fagnes - Belgium). Climate of the past 9, 2889-2928. http://hdl.handle.net/2268/155396

Allan et al. 2015. Lead concentrations and isotope ratios in speleothems as proxies for atmospheric metal pollution since the Industrial Revolution. Chemical Geology, http://hdl.handle.net/2268/178711

Allan et al. 2018. Evidence for solar influence in a Holocene speleothem record (Pere Noel cave, SE Belgium). Quaternary Science Reviews $192 \quad$ (2018) 249-262. http://hdl.handle.net/2268/224568

Bradley, R. 1999. Paléoclimatology. Intern. Geophys. Series vol. 64. Academic Press. San diego. USA.

Oberlin, C. 2005. Dossier d'archéologie. Le Carbone 14 et ses apports à l'archéologie.

Walker, M. 2005. Quaternary dating methods. Wiley \& Sons, Chichester, England. 